\title{
Fictionalism and the incompleteness problem
}

\author{
Lukas Skiba ${ }^{1}$
}

Received: 27 April 2015 / Accepted: 18 December 2015 / Published online: 19 January 2016

C The Author(s) 2016. This article is published with open access at Springerlink.com

\begin{abstract}
Modal fictionalists face a problem that arises due to their possible-world story being incomplete in the sense that certain relevant claims are neither true nor false according to it. It has recently been suggested that this incompleteness problem generalises to other brands of fictionalism, such as fictionalism about composite or mathematical objects. In this paper, I argue that these fictionalist positions are particularly threatened by a generalised incompleteness problem since they cannot emulate the modal fictionalists' most attractive response. I then defend mathematical and compositional fictionalism by showing that the reasons for which the incompleteness problem has been thought to affect them are mistaken. This leads to the question of whether there are other fictionalist positions to which the problem does in fact generalise. I give a general account of the features of a fictionalist position that generate the incompleteness problem and argue that whenever a fictionalist position does exemplify these features then the problem can be addressed in analogy to the modal fictionalists' preferred response.
\end{abstract}

Keywords Fictionalism · Possible worlds · Mathematical objects ·

Composite objects $\cdot$ Fictional incompleteness

\section{Modal fictionalism and the incompleteness problem}

Modal realists endorse all instances of the following schema

(R-Mod)

$$
\phi \leftrightarrow[\phi]_{T}
$$

where $\phi$ is an ordinary modal sentence and $[\phi]_{T}$ is $\phi$ 's regimentation in the realists'

\footnotetext{
$凶 \quad$ Lukas Skiba

lps24@cam.ac.uk

1 Faculty of Philosophy, University of Cambridge, Sidgwick Avenue, Cambridge CB3 9DA, UK
} 
possible-world language. Following Rosen (1990), modal fictionalists want to exploit the benefits of the realists' possible-world language without incurring ontological commitment to any possible worlds other than the actual one. To do so, they replace the realists' schema (R-Mod) with the following schema:

(F-Mod)

$$
\phi \leftrightarrow \text { According to } P W,[\phi]_{T}
$$

Here, the right-hand side is what we might call a meta-fictional sentence which says of the possible-world fiction $P W$-standardly taken to be some suitable formulation of modal realism - that the sentence $[\phi]_{T}$ holds according to it. Modal fictionalists now face a problem that arises from the fact that modal realism - and consequently the possible-world fiction $P W$-is incomplete. The standard example relates to Lewis's recombination principle. ${ }^{1}$ Following Rosen (1990, p. 333), we can spell out this principle as follows:

(RP) For any collection of concrete objects from any number of worlds, there is a single world containing any number of non-overlapping duplicates of each, provided there is a spacetime large enough to hold them.

The crucial aspect is the unspecific restriction after the last comma. It is a restriction because it ensures that the principle doesn't allow for the combination of any number of concrete non-overlapping objects in a single world, but requires the number to fall below an upper bound to be determined by the maximum possible size of spacetime. The restriction is unspecific, because despite asserting the existence of such an upper bound $\lambda$ and locating $\lambda$ within the infinite cardinals, Lewis explicitly intends modal realism to be agnostic about exactly which cardinal $\lambda$ is (cf. 1986, Sects. 1.8, 2.2).

Now, let $\kappa$ be some infinite cardinal larger than the number of concrete nonoverlapping objects that actually exist and let $[k]_{T}$ be the sentence of the possible-world language that says that there is a world containing $\kappa$ objects. Then consider the sentence:

$$
\text { According to } P W,[k]_{T}
$$

On the face of it, (1) is false. It erroneously asserts that $P W$ endorses the existence of a world with $\kappa$ objects. But in fact $P W$ does no such thing. For whether there is such a world according to $P W$ depends on whether $\kappa \leq \lambda$. And since $P W$ is silent on exactly which number $\lambda$ is, it must consequently also be silent on whether there is a world with $\kappa$ objects. So (1) is false, and its negation is true:

$$
\left.\neg \text { (According to } P W,[k]_{T}\right)
$$

But now consider:

$$
\text { According to } P W,[\neg k]_{T}
$$

If (1) is false, then so is (2). $P W$ doesn't assert the existence of a world with $\kappa$ objects, but it doesn't rule out its existence either. Thus we also have:

\footnotetext{
${ }^{1}$ See for instance Rosen (1990, Sect. 7), Chihara (1998, pp. 175-177), Sider (2002, p. 314), Fine (2003, p. 117); Brogaard (2006, p. 81), Nolan (2011, Sect. 4.2), Woodward (2012, p. 782)
} 
$(\neg 2)$

$\neg\left(\right.$ According to $\left.P W,[\neg k]_{T}\right)$

The conjunction of the sentences $(\neg 1)$ and $(\neg 2)$ constitutes what we will call an incompleteness sentence for modal fictionalists:

(Inc-Mod) $\quad \neg$ (According to $\left.P W,[k]_{T}\right) \wedge \neg$ (According to $P W,[\neg k]_{T}$ )

Taken by itself, such an incompleteness sentence is unproblematic. Modal realists, for example, can happily accept it. It is only in the hands of the modal fictionalists that (Inc-Mod) becomes problematic. For the following are instances of the fictionalist schema (F-Mod):

(F-Mod-1) $\quad k \leftrightarrow$ According to $P W,[k]_{T}$

(F-Mod-2) $\quad \neg k \leftrightarrow$ According to $P W,[\neg k]_{T}$

And in tandem with (Inc-Mod) these instances entail a contradiction:

(Con-Mod)

$\neg k \wedge \neg \neg k$

The incompleteness problem for modal fictionalism thus consists in the fictionalists' being forced to march from incompleteness to inconsistency via two instances of their schema.

\section{The threat of a general incompleteness problem}

Fictionalism about possibilia is just one instance of a general fictionalist strategy. Along similar lines, mathematical fictionalists (following Field 1980, 1989) try to eschew numbers and sets, while compositional fictionalists (following Dorr and Rosen 2002) seek to avoid commitment to molecules and tables. It is thus not surprising that certain problems which are first discovered in the context of a particular fictionalism subsequently prove to affect an entire family of fictionalist positions. For example, the Brock-Rosen objection (Brock 1993; Rosen 1993), originally directed at modal fictionalism, was found to be a structural problem encountered by a variety of fictionalisms (Nolan and O'Leary-Hawthorne 1996). It is thus a natural question to ask whether the modal fictionalists' incompleteness problem also generalises to other brands of fictionalism. Woodward (2012) proposes a positive answer to this question. In particular, he suggests that the problem affects mathematical and compositional fictionalists, since certain theories-upon which they might want to base their fictions-give rise to incompleteness sentences.

In the case of mathematical fictionalism, Woodward considers fictionalists about sets who choose Zermelo-Fraenkel set theory $(Z F)$ as their fiction of choice. $Z F$ leaves the continuum hypothesis unsettled, so that if we let $[c]_{T}$ be the regimentation of this hypothesis into the language of $Z F$ we have:

(Inc-Math) $\quad \neg$ (According to $\left.Z F,[c]_{T}\right) \wedge \neg$ (According to $Z F,[\neg c]_{T}$ )

What about compositional fictionalists? According to Woodward, they are likely to face an incompleteness problem too, but this time he doesn't provide us with an example of an incompleteness sentence. If we think of the relevant fiction as endorsing an entirely unrestricted principle of composition, examples are indeed hard to come 
by. But compositional fictionalists might well be interested in basing their composition story, $C S$, on a principle that is closer to common sense than a principle of unrestricted composition. Such a principle will say that some given objects compose another object only if they fulfil a certain condition. For example, this condition might be that the objects are suitably spatio-temporally cohesive. Now suppose there are two objects, say a cup and a saucer, which neither clearly meet the composition condition nor clearly fail to meet it. Let $[o]_{T}$ be the $C S$-regimentation of the claim that there is an object composed of the cup and the saucer. It now seems that $C S$ neither endorses $[o]_{T}$ nor its negation, so that we can construct an incompleteness sentence for this fiction too:

(Inc-Comp) $\quad \neg$ (According to $\left.C S,[o]_{T}\right) \wedge \neg$ (According to $C S,[\neg o]_{T}$ )

Given that we can replicate incompleteness sentences for mathematical and compositional fictionalism a general incompleteness problem doesn't seem far fetched. What is more, I submit that if the problem does indeed affect mathematical and compositional fictionalism, it will be more recalcitrant in these cases than in the case of modal fictionalism. This is because one of the more attractive responses to the problem available to modal fictionalists proves unavailable to their mathematical and compositional counterparts.

The response I have in mind has been first proposed by Fine (2003) and has since found approval in Brogaard (2006) and Nolan (2011, supplement to Sect. 4.2). It starts from the observation that, for all its benefits, modal fictionalism is now widely regarded as not allowing for a reduction of the modal to the non-modal (see for instance Nolan 1997). The main idea is then that non-reductive modal fictionalists can invoke modal material in specifying the content of their fiction in a way that ensures the fiction's completeness.

We can spell out this modal content response as follows. In a first step, non-reductive modal fictionalists can regard $P W$ as straightforwardly endorsing the realists' schema:

(R-Mod)

$$
\phi \leftrightarrow[\phi]_{T}
$$

When conceived of in this way, $P W$ thus contains the two following biconditionals:

(R-Mod-1)

(R-Mod-2)

It is possible that there are $\kappa$ objects $\leftrightarrow$ there is a world that contains $\kappa$ objects

It is not possible that there are $\kappa$ objects $\leftrightarrow$ there is no world that contains $\kappa$ objects

In a second step, non-reductive modal fictionalists can incorporate all the ordinary modal truths into their fiction. Since there are infinitely many such truths they cannot, of course, incorporate them into the fiction's explicit content by writing them all down. But then they needn't. As Lewis points out we are familiar with regarding the content of a fiction as a joint product of its explicit content and a background of genuine truths carried over into the fiction 'not because there is anything explicit in the fiction to make them true, but rather because there is nothing to make them false' (1978, p. 42). The fictionalists can thus regard the modal truths as part of the background of facts implicitly carried over into the content of $P W$ in the same way in which a truth such 
as 'water is $\mathrm{H}_{2} \mathrm{O}$ ' is implicitly carried over into the content of the Holmes-stories. ${ }^{2}$ $P W$ will then contain either

(R-Mod-1a) It is possible that there are $\kappa$ objects.

or

(R-Mod-2a) It is not possible that there are $\kappa$ objects.

depending on which of these sentences in fact happens to be true.

It should be clear that once $P W$ is conceived of in this way, it is guaranteed to be immune to the incompleteness problem. If in fact (R-Mod-1a) is true then it will be part of the fiction. Since the fiction also contains the biconditional (R-Mod-1), it will also endorse

(R-Mod-1b) There is a world that contains $\kappa$ objects

in which case the left conjunct of (Inc-Mod) is false:

(Inc-Mod) $\quad \neg$ (According to $\left.P W,[k]_{T}\right) \wedge \neg$ (According to $P W,[\neg k]_{T}$ )

If, on the other hand, (R-Mod-2a) is true then the fiction will by the same reasoning endorse

(R-Mod-2b) There is no world that contains $\kappa$ objects

thus rendering the right conjunct of (Inc-Mod) false. Either way the incompleteness sentence is guaranteed to be false and the problem is thereby avoided.

Now it is hard to see how mathematical and compositional fictionalists could deal with incompleteness in an analogous way. For note that the modal content response exploits the fact that the term 'modal fictionalism' is somewhat misleading. Modal fictionalists reject possible worlds; they don't reject modality. This is why we could advise them to include all the ordinary modal truths into the content of their fiction,

\footnotetext{
${ }^{2}$ Note that while modal fictionalists can appeal to the general phenomenon of background facts being implicitly carried over into fictions to motivate this step, they cannot straightforwardly appeal to Lewis's (1978) particular account of this phenomenon which invokes possible worlds. This, however, should not be held against the modal content response. For even modal fictionalists who don't go in for the modal content response will find themselves in exactly the same situation. To see this, compare Rosen's (1990, Sect. 3) original conception of $P W$ with the present conception.

On Rosen's conception, $P W$ contains Lewis's recombination principle: (RP) from Sect. 1. As Rosen acknowledges, (RP) is itself insufficient to generate possible world sentences. To do so, (RP) needs to be combined with what Rosen calls an Encyclopedia: 'a list of the non-modal truths about the intrinsic character of this universe' (p. 335). The idea is that by incorporating the Encyclopedia into $P W$ we ensure that $P W$ recognizes the existence of a suitable 'starter kit' of objects from which all the required possibila can be generated by recombination.

In comparison, on the present way of spelling out the modal content response $P W$ contains the realists' schema (R-Mod), which itself is insufficient to generate the required possible world sentences. To do so, (R-Mod) needs to be combined with a list of the modal truths (call this list the Compendium).

Now, what holds of our Compendium equally holds of Rosen's Enyclopedia: a 'list of the non-modal truths about the intrinsic character of this universe' cannot be explicitly included into $P W$ any more than a list of the modal truths. So whether or not modal fictionalists go in for the modal content response, they will have to rely on the idea that a large body of truths can be implicitly incorporated into $P W$ 's content, without being able to explain this phenomenon in terms of Lewis's possible world account. Thanks to an anonymous reviewer for pressing me to clarify this issue.
} 
so that these truths, in tandem with the realist schema, could generate the required possible world sentences. In the case of mathematical and compositional fictionalism, the analogous move is unavailable. For instance, compositional fictionalists maintain that, in fact, there aren't any composite objects. So they can't make their composition story complete by incorporating all the compositional truths into it. For if they are right about what these truths are, then doing this would just turn the fiction into a testimony of their compositional nihilism and thereby render it useless for their purposes. The same applies mutatis mutandis to mathematical fictionalists, so that they cannot easily complete their fiction either.

With the generalisation of the modal content response having failed, it might be hoped that there is another, similarly attractive response available to the modal fictionalist which can furthermore be generalised to other brands of fictionalism. Nolan (2011) suggests that fictionalists can avoid the incompleteness problem by restricting their schema (F-Mod) rather than by ensuring the fiction's completeness. ${ }^{3}$ In particular, Nolan suggests replacing the fictionalist schema with the following (2011, supplement to Sect. 4.2):

\section{(F-Mod-Res)}

$$
\phi \leftrightarrow \text { According to } P W,[\phi]_{T}
$$

(unless $P W$ is silent about $[\phi]_{T}$, in which case $\phi$ is truth-valueless)

It is clear that retreating from (F-Mod) to (F-Mod-Res) blocks the derivation of (ConMod) from (Inc-Mod). However, avoiding the incompleteness problem in this way is not in fact advisable to modal fictionalists.

For one thing, Nolan's particular suggestion has it that the ordinary modal sentence $\phi$ is truth-valueless whenever the story is incomplete with regard to the corresponding possible world sentence $[\phi]_{T}$. Nolan's restriction response is thus committed to non-classical truth-values (and, accordingly, to non-classical connectives which render conjunctions with truth-valueless conjuncts false, and disjunctions with truth-valueless disjuncts true). In this regard it is very similar to Rosen's (1990, Sect. 7) original response to the incompleteness problem which makes do without a restriction of the schema at the cost of regarding not only the modal sentence $\phi$ but also the corresponding meta-fictional sentence 'According to $P W,[\phi]_{T}$ ' as truth-valueless whenever the fiction is silent on $[\phi]_{T}$.

In virtue of this similarity, Nolan's restriction response inherits one of the main problems of Rosen's original proposal. As Rosen admits, invoking non-classical machinery appears hopelessly ad hoc if this is done for the mere purpose of fixing a bug in the fictionalist's theory. Such a move might be justifiable in an attempt to account for general semantic phenomena such as vagueness or the liar paradox. But in the present case the fictionalist is applying it to discourse where 'our ordinary ways of thinking give us no reason to expect a complicated propositional logic, and where the only reason for proposing one is, to say the least, generated by concerns rather distant from the linguistic practice in question' (Rosen 1990, p. 343).

Now Nolan's claim that $\phi$ is truth-valueless when the story is silent on $[\phi]_{T}$ doesn't appear essential to the restriction strategy. The fictionalist could impose the restriction as before but maintain that when the story is silent on $[\phi]_{T}$ then $\phi$ and $\neg \phi$ receive

\footnotetext{
${ }^{3}$ Thanks to an anonymous referee for prompting the ensuing discussion of the restriction strategy.
} 
opposite classical truth-values, although we might not be able to tell which sentence receives which truth-value. This way the restriction strategy dodges the charge of making an ad hoc appeal to non-classical resources. This is not to say that the strategy doesn't still appear somewhat ad hoc or at least inelegant. But in fact there is a much more significant reason to reject even this improved restriction strategy.

One of the main reasons why modal fictionalists want to preserve the right to talk in possible world terms is that it enables them to do 'modal logic by proxy' (Divers 1999 , Sect. V). That is, when confronted with an argument couched in modal terms, $\psi \therefore \phi$, fictionalists want to consider the corresponding argument couched in possible world terms, $[\phi]_{T} \therefore[\psi]_{T}$, and will want to take the validity of the latter to reveal the validity of the former. Apart from making inferences more tractable, this is taken to have an advantage of conceptual economy: the validity of an argument couched in modal language reduces to the validity of an argument in the first order language of counterpart theory (cf. Divers 1999, p. 329; Woodward 2010, p. 410).

As Divers points out, in order for fictionalists to be justified in doing modal logic by proxy, they had better be able to establish the following principle:

(Safety)

If the $P W$-sentence $[\phi]_{T}$ entails the $P W$-sentence $[\psi]_{T}$, then the modal sentence $\phi$ entails the modal sentence $\psi$.

Fortunately, Divers has also shown how (Safety) can be established (1999 Sect. VI, see also Woodward 2010, Sect. 2). In brief, the strategy is as follows. Given a modal conception of entailment, the task is that of establishing the strict conditional $\square(\phi \rightarrow$ $\psi)$ on assumption of $\square\left([\phi]_{T} \rightarrow[\psi]_{T}\right)$. To do this we assume $\phi$ for conditional proof. By appealing to the fictionalist biconditional (F-Mod) we derive 'According to $P W$, $[\phi]_{T}$ '. This, together with $\square\left([\phi]_{T} \rightarrow[\psi]_{T}\right)$, yields 'According to $P W,[\psi]_{T}$ ' on the assumption that the story operator is closed under entailment. By appealing again to (F-Mod) we derive $\psi$. Discharging the assumption of $\phi$, this yields $\phi \rightarrow \psi$. To arrive at the desired necessitation of this conditional, Divers argues that the fictionalist is entitled to assume the necessitations of all the assumption appealed to in the proof. This guarantees the truth of $\square(\phi \rightarrow \psi)$ given the principle (which holds in any system at least as strong as $S 4$ ) that whenever $\psi$ follows from $\phi_{1}, \ldots, \phi_{n}$, then $\square \psi$ follows from $\square \phi_{1}, \ldots, \square \phi_{n}$.

Now the problem for the restriction strategy is this: modal fictionalists will want to be able to do modal logic by proxy even when confronted with a modal argument which contains a sentence whose possible world paraphrase is not settled by the fiction. Just because we don't know whether the modal sentence $k$ is true (i.e. don't know whether it is possible that there are $\kappa$ many individuals) doesn't mean that we should not be interested in the validity of arguments which contain $k$ as a premiss or as conclusion. Quite the contrary, evaluating such arguments might well play an important part in coming to know whether the sentence is true. But a fictionalist who employs the restriction strategy won't be able to justify the practice of doing modal logic by proxy when it comes to any such argument. As we have seen the justification of this practice requires establishing (Safety) for the relevant argument. And establishing (Safety) for an argument that features the modal sentence $k$ will require invoking ' $k \leftrightarrow$ According to $P W,[k]_{T}$ ' which is derivable only from the general schema (F-Mod), not by the restricted schema (F-Mod-Res). 
Unless modal fictionalists are prepared to give up the right to do modal logic by proxy in some important cases, they are thus well advised to address the incompleteness problem in a way that preserves the fictionalist schema (F-Mod). As we have seen, the modal content response is a simple and attractive way to do this. It is not the only way. We have already mentioned Rosen's original proposal with its commitment to non-classical truth-values for both modal and meta-fictional sentences. A further alternative is provided by Woodward (2012) whose solution to the incompleteness problem commits him to an analysis of the story operator in terms of counterfactual conditional that supports counterfactual excluded middle (p. 785), and involves regarding modal sentences such as $k$ as either truth-valueless, metaphysically indeterminate or in principle unknowable (pp. 788-789). What makes the modal content response so attractive is that it makes do without such controversial commitments.

Unfortunately, we have seen that the modal content response doesn't generalise to mathematical and compositional fictionalism. A general incompleteness problem thus has the potential to seriously upset these fictionalists. But not only them. If the problem affects compositional and mathematical fictionalists, this will also indirectly affect modal fictionalists. For while they can ensure their fiction's completeness via the modal content response, this response will appear much less attractive if the problem also arises for their compositional and mathematical brethren who cannot react to it in an analogous way. A general problem, it might reasonably be said, requires a general solution, so that the modal content response, though effective in the particular case for which it was designed, will appear less satisfactory due to its limited scope.

\section{Undermining the threat for mathematical and compositional fictionalism}

Given the explosive nature of a positive answer to the generalisation question, fictionalists are well advised to have a closer look at the precise reasoning given in support of it. In the previous section we agreed with Woodward that mathematical and compositional fictionalism are prone to incompleteness sentences. But this falls short of establishing that these positions face an incompleteness problem. For taken by themselves (Inc-Math) and (Inc-Comp) are no more problematic than (Inc-Mod) was. In the case of modal fictionalism, remember, it was only the combination of (IncMod) with the modal fictionalist schema (F-Mod) that gave rise to the problem. So what are the analogous schemas that mathematical and compositional fictionalists are supposed to endorse and whose instances are supposed to give rise to a contradiction when combined with the relevant incompleteness sentence?

To understand Woodward's answer, we must first get clear on his interpretation of the modal fictionalists' schema:

(F-Mod) $\quad \phi \leftrightarrow$ According to $P W,[\phi]_{T}$

So far we have taken the modal fictionalists' position to be exhaustively characterised by their endorsement of what we may call the the modal instances of this schema: the instances that result from replacing $\phi$ with an ordinary modal sentence. This, however, seems insufficient to achieve one of their central ambitions. For besides providing a deflationary account of ordinary modal discourse, an important aim of 
modal fictionalism has always been to enable guilt-free indulgence in unqualified possible world talk. Endorsing all and only the modal instances of (F-Mod) reflects the former aim but doesn't help with the latter. To remedy this shortcoming, Liggins (2008) suggests that the modal fictionalists' schema should not be restricted to ordinary modal sentences. Rather it should also be seen as providing an ontologically harmless semantics for sentences explicitly quantifying over possible worlds. According to this account, modal fictionalists not only endorse the modal instances of (F-Mod) but additionally assert what we may call its worldly instances: the instances which result from replacing $\phi$ with a sentence explicitly quantifying over possible worlds, such as (F-Mod-3)

There is a possible world that contains a blue swan $\leftrightarrow$ According to $P W$, there is a possible world that contains a blue swan.

Importantly, when it comes to such worldly instances of the schema, fictionalists are taken to give the right-hand side 'semantic priority' (Liggins 2008, p. 153). That is, in accordance with their anti-realism about possible worlds, when fictionalists endorse such a worldly instance they take the left-hand side to express the ontologically harmless meta-fictional proposition more perspicuously expressed by the right-hand side.

Now, Woodward (2012, p. 782, fn. 2; 2008, Sect. 2) follows Liggins in this interpretation of the fictionalist schema in all important respects. ${ }^{4}$ And he takes mathematical and compositional fictionalists to similarly offer an ontologically harmless semantics for talk about mathematical and compositional objects. That is, he takes them to endorse all those biconditionals that result from the relevant schema by replacing $\phi$ with a sentence explicitly quantifying, respectively, over sets or composite objects:

(F-Math) $\quad \phi \leftrightarrow$ According to $Z F,[\phi]_{T}$

(F-Comp) $\quad \phi \leftrightarrow$ According to $C S,[\phi]_{T}$

Thus according to Woodward, the compositional fictionalist endorses, for example, the following instances of (F-Comp), where $o$ is the claim that there is an object composed of the cup and the saucer:

(F-Comp-1) $\quad o \leftrightarrow$ According to $C S,[o]_{T}$

(F-Comp-2) $\quad \neg o \leftrightarrow$ According to $C S,[\neg o]_{T}$

And it is these instances which Woodward takes to give rise to a contradiction when combined with the incompleteness sentence for $C S$ :

(Inc-Comp) $\quad \neg$ (According to $\left.C S,[o]_{T}\right) \wedge \neg$ (According to $C S,[\neg o]_{T}$ )

After all this trio allows us to infer

(Con-Comp) $\quad \neg O \wedge \neg \neg O$

\footnotetext{
4 Woodward's presentation of the fictionalist position differs from Liggins's in that he formulates the fictionalist schemas in a metalinguistic fashion: ' $\phi$ ' is true $\leftrightarrow$ According to THE FICTION, $[\phi]_{T}$. This difference is not important for the present discussion; the reason for which the incompleteness problem doesn't carry over to mathematical and compositional fictionalism is not sensitive to the choice between the standard version of the schema and its meta-linguistic variant.
} 
At this point it might thus appear that compositional fictionalists indeed find themselves marching from incompleteness to inconsistency. Of course, analogous reasoning will have mathematical fictionalists join them on this unpleasant journey.

I believe, however, that closer inspection will reveal that we are dealing with a fictional problem rather than a problem for fictionalism. For although (Con-Comp) might look contradictory, in fact it isn't. To see this, first consider the left conjunct of (Con-Comp), the sentence $\neg o$, which is derived from the left conjunct of (IncComp) together with (F-Comp-1). And remember that $o$ is the sentence 'there is an object composed of the cup and the saucer' which explicitly quantifies over composite objects. The compositional fictionalist can only be seen as endorsing (F-Comp-1) given that the right-hand side is taken to enjoy semantic priority. For if $o$, as it occurs on its left-hand side, were taken at face value, rather than as expressing the metafictional proposition that is more perspicuously expressed by the right-hand side, asserting this instance would undermine the compositional fictionalists' anti-realism about composite objects.

But then we must of course keep this fact in mind when we use (F-Comp-1) in our inference. So when we combine (Inc-Comp) and (F-Comp-1) to derive the negation of (F-comp-1)'s left-hand side, $\neg o$, we must keep in mind that here the negated sentence, $o$, really expresses the proposition more perspicuously expressed by 'According to $C S$, $[o]_{T}$ '. But then then the derived sentence, $\neg o$, expresses the negation of that proposition, i.e. it expresses the proposition more perspicuously expressed by ' $\neg$ (According to $\left.C S,[o]_{T}\right)^{\prime}$.

So, compositional fictionalists are indeed committed to the sentence that constitutes the left conjunct of (Con-Comp). But from their perspective the proposition expressed by this sentence can be more perspicuously expressed by the negation of the right-hand side of (F-comp-1):

\section{$\neg\left(\right.$ According to $\left.C S,[o]_{T}\right)$}

It should now be clear that commitment to (Con-Comp) is unproblematic. Analogous reasoning, when applied to the derivation of the right conjunct of (Con-Comp), the sentence $\neg \neg O$, shows that this sentence must really be taken to express the proposition more perspicuously expressed by the negation of the right-hand side of (F-comp-2):

$$
\neg\left(\text { According to } C S,[\neg o]_{T}\right)
$$

So if we keep in mind the assumptions we have to make in order to derive (Con-Comp), we can see this sentence doesn't really express a contradictory proposition. In fact, (Con-Comp) just expresses the proposition more perspicuously expressed by:

\section{(Con-Comp*) $\neg$ (According to $\left.C S,[o]_{T}\right) \wedge \neg\left(\right.$ According to $\left.C S,[\neg o]_{T}\right)$}

And this is nothing but the harmless incompleteness sentence we started out with. Accepting the incompleteness sentence (Inc-Comp) thus doesn't lead compositional fictionalists to a contradiction.

On Woodward's account, the mathematical fictionalists' take on (F-Math) is just the same as the compositional fictionalists' take on (F-Comp). The argument thus equally applies to them, showing that mathematical fictionalists don't end up being committed to a contradictory proposition as a result of accepting (Inc-Math) either. For the same 
reason the argument also shows that it is only the modal instances, and not the worldly instances, that generate the problem in the case of modal fictionalism.

The upshot of this is that even if the compositional or mathematical fictionalists' stories give rise to incompleteness sentences, Woodward's considerations don't in fact provide any reason to believe that they face an incompleteness problem.

Accordingly, there is no need for these fictionalists to reject the incompleteness sentences (Inc-Comp) and (Inc-Math). This is good news for compositional and mathematical fictionalists, since we have seen that they would have been hard-pressed to do so with the most attractive defensive manoeuvre available to the modal fictionalists, the modal content response, being unavailable to them.

\section{Undermining the threat in general}

We have seen that the incompleteness problem doesn't arise for mathematical and compositional fictionalists in the way suggested by Woodward. Can it still arise for these or other types of fictionalism in some other shape or form? We are now in a better position to see which structural features of a fictionalist position are responsible for the problem's arising and this will enable us to arrive at a more general conclusion.

The discussion in the previous section shows that the following two features of a fictionalist position, based on a given fiction $f$, are not sufficient for this position to face an incompleteness problem:

Fiction $f$ gives rise to an incompleteness sentence: $\neg\left(\right.$ According to $\left.f,[p]_{T}\right) \wedge \neg\left(\right.$ According to $\left.f,[\neg p]_{T}\right)$

The fictionalist subscribes to a schema with the following instances: $p \leftrightarrow$ According to $f,[p]_{T}$ $\neg p \leftrightarrow$ According to $f,[\neg p]_{T}$

Instead, the problem only arises if the position additionally has the following feature:

The fictionalists do not assign semantic priority to the right-hand side of the fictionalist schema. That is, they do not regard the left-hand sides as expressing the meta-fictional propositions more perspicuously expressed by the right-hand sides.

The reason why the incompleteness problem doesn't arise for mathematical and compositional fictionalism as conceived by Woodward is that they exhibit the first two features but not the third. And this isn't an artifact of Woodward's presentation. These fictionalists could only be portrayed as endorsing the relevant instances of the schemas (F-Comp) and (F-Math) — without thereby undermining their fictionalist credentialsbecause it had been ensured that they assign semantic priority to the right-hand sides of these schemas.

In contrast, some-but not all-modal fictionalist positions will satisfy all three conditions and will thus be susceptible to the incompleteness problem. Following Nolan (1997) we can distinguish between timid and strong modal fictionalists. Both camps endorse all the instances of the schema (F-Mod) which result from replacing $\phi$ with an ordinary modal sentence. But strong modal fictionalists additionally 
take the content of the ordinary modal sentence to coincide with the meta-fictional proposition expressed by the right-hand side. Timid modal fictionalists in contrast, endorse the schema but take the modal sentences at face value, i.e. take these sentences to express propositions which are distinct from the meta-fictional propositions expressed by the right-hand sides of the schema. In doing so they exemplify feature (iii). It is thus only timid modal fictionalism which exhibits the three features that generate the incompleteness problem. Strong modal fictionalism, on the other hand, will take modal instances of (F-Mod) to have just the same content as the corresponding worldly instances. And we have seen in the previous section that those instances don't generate an inconsistency. In this regard, the incompleteness problem thus turns out to be less general than anticipated, since it doesn't even arise on all varieties of modal fictionalism.

Is there room (and motivation) for other fictionalisms to endorse a schema in a fashion relevantly similar to the timid modal fictionalists' endorsement of (F-Mod)? That is, might some fictionalists wish to tie the truth-values of the sentences in a certain domain of discourse (analogous to the ordinary modal sentences) via some schema (analogous to F-Mod) to meta-fictional sentences (analogous to the 'According to $P W^{\prime}$-sentences) while taking the original sentences at face value?

Perhaps. Consider presentists who refuse to accept the existence of any times other than the present. Presentists might still wish to join their eternalist opponents in spelling out the truth-conditions for sentences containing tense operators, such as 'it was the case that there exists a dinosaur', in terms of quantifications over past times, as in 'there is a time $t$ earlier than the present time such that at $t$ there exists a dinosaur'. But, qua presentists, they will want to do so without being ontologically committed to such non-present times. To this end, the presentists might consider adopting a fictionalist attitude towards eternalism $(E T) .{ }^{5}$ This will involve rejecting the eternalists' schema ${ }^{6}$

(R-Time) $\quad \phi \leftrightarrow[\phi]_{T}$

and replacing it with the fictionalist schema

(F-Time) $\quad \phi \leftrightarrow$ According to $E T,[\phi]_{T}$

Such temporal fictionalists might well be timid in that they continue to take the tensed sentences at face value rather than as expressing meta-fictional propositions. This fictionalist position will thus exemplify features (ii) and (iii). Whether it faces an incompleteness problem accordingly depends on whether an incompleteness sentence can be constructed for the eternalist fiction. However, we are now in a position to see that even if this were the case, it shouldn't cause too much concern. In Sect. 2 we saw that what made the possibility of a generalised incompleteness problem so menacing was the worry that the most attractive response available to modal fictionalists wouldn't generalise in step. Having acquired a better understanding of the structural features responsible for the problem, we can now dispel this worry.

\footnotetext{
${ }^{5}$ Combining presentism with a fictionalist attitude towards eternalism is contemplated by Sider (1999, p. 21, n. 30).

${ }^{6}$ Similar to the modal realists' schema (R-Mod), $\phi$ is an ordinary tensed sentence and $[\phi]_{T}$ is $\phi$ 's regimentation in the eternalists' time-committing language.
} 
As we've seen, the modal fictionalists can guarantee their fiction's completeness by incorporating all the modal facts into its content. We can now see that this works precisely because they can adopt a timid attitude towards their schema, i.e. because they can, without undermining their fictionalism about worlds, regard ordinary modal sentences as expressing truths when taken at face value.

Now the timid temporal fictionalists will also be able to employ this strategy. They can, without undermining their fictionalism about times, regard tensed sentences, such as 'it was the case that there exists a dinosaur', as true while taking them at face value. And this allows them to embrace a tensed content response in analogy to the modal content response: they can ensure the fiction's completeness by incorporating all the ordinary tensed truths into its content. This is no coincidence. The very structural similarity to timid modal fictionalism that made timid tensed fictionalism susceptible to the incompleteness problem at the same time ensures that the modal fictionalists' preferred response strategy can be carried over: the incompleteness problem can only arise when the fictionalists connect a range of target sentences with meta-fictional sentence while taking the target sentences at face value, thus exhibiting features (ii) and (iii). But it is precisely these features which in turn allow them to ensure the fiction's completeness via incorporating the relevant truths - those expressed by the target sentences when taken at face value-into their fiction, thereby making sure that feature (i) is not instantiated.

The incompleteness problem, then, may not be localised to timid modal fictionalism. But it can only generalise to fictionalisms whose structural similarity to timid modal fictionalism ensures that the problem can be addressed in a way analogous to the modal fictionalists' preferred response.

Acknowledgements I am grateful to Arif Ahmed, Tim Button, Adam Caulton, Fiona Doherty, Owen Griffiths, Mirja Holst, David Liggins, Alex Oliver, Shyane Siriwardena, Rob Trueman, Nathan Wildman, and the anonymous referees from Synthese for helpful comments. This work was presented at the Serious Metaphysics Group (Cambridge), the Joint Sessions 2014 (Cambridge), the 8th European Conference of Analytic Philosophy (Bucharest), and the Issues on the (Im)possible II Conference (Bratislava). I would like to thank the organisers and audiences for helpful discussion and in particular Daniel Dohrn for giving a response to this paper at the last mentioned conference. Special thanks to Richard Woodward for providing detailed written comments on an earlier version of this paper. This research was supported by the Robert Smithson Studentship from the Faculty of Philosophy at the University of Cambridge and by an Analysis Studentship from the Analysis Trust.

Open Access This article is distributed under the terms of the Creative Commons Attribution 4.0 International License (http://creativecommons.org/licenses/by/4.0/), which permits unrestricted use, distribution, and reproduction in any medium, provided you give appropriate credit to the original author(s) and the source, provide a link to the Creative Commons license, and indicate if changes were made.

\section{References}

Brock, S. (1993). Modal fictionalism: A response to Rosen. Mind, 102, 147-150.

Brogaard, B. (2006). Two modal-isms: Fictionalism and eratzism. Philosophical Perspectives, 20, 77-94. Chihara, C. (1998). The worlds of possibility. Oxford: Clarendon Press.

Divers, J. (1999). A modal fictionalist result. Nous, 33, 317-346.

Dorr, C., \& Rosen, G. (2002). Composition as a Fiction. In R. Gale (Ed.), The Blackwell guide to metaphysics (pp. 151-174). Oxford: Blackwell.

Field, H. (1980). Science without numbers: A defense of nominalism. Oxford: Blackwell. 
Field, H. (1989). Realism, mathematics and modality. Oxford: Blackwell.

Fine, K. (2003). The problem of possibila. In M. J. Loux \& D. W. Zimmerman (Eds.), Oxford handbook of metaphysics (pp. 161-179). Oxford: Oxford University Press.

Lewis, D. (1978). Truth in fiction. American Philosophical Quarterly, 15, 37-46.

Lewis, D. (1986). On the plurality of worlds. Oxford: Blackwell.

Liggins, D. (2008). Modal fictionalism and possible-worlds discourse. Philosophical Studies, 138, 151-160.

Nolan, D. (1997). Three problems for "strong" modal fictionalism. Philosophical Studies, 87, 259-275.

Nolan, D. (2011). Modal fictionalism. In E. Zalta (Ed.), The stanford encyclopaedia of philosophy (Winter 2011 Edition), from http://plato.stanford.edu/archives/win2011/entries/fictionalism-modal/.

Nolan, D., \& O’Leary-Hawthorne, J. (1996). Reflexive fictionalisms. Analysis, 56, 23-32.

Rosen, G. (1990). Modal fictionalism. Mind, 99, 327-354.

Rosen, G. (1993). A problem for fictionalism about possible worlds. Analysis, 53, 71-81.

Sider, T. (1993). Presentism and ontological commitment. Journal of Philosophy, 96, 325-347.

Sider, T. (2002). The ersatz pluriverse. Journal of Philosophy, 99, 279-315.

Woodward, R. (2008). Why modal fictionalism is not self-defeating. Philosophical Studies, 139, $273-288$.

Woodward, R. (2010). Modal fictionalism and inferential safety. Analysis, 70, 409-417.

Woodward, R. (2012). Fictionalism and incompleteness. Nous, 46, 781-790. 